\title{
The application of near-infrared spectra micro-image in the imaging analysis of biology samples
}

\author{
Dong Wang*, ${ }^{\star}$, , Yun-Sheng Ding ${ }^{\dagger}$, Zhong-Hua Guo* \\ and Shun-Geng Min*, \\ *Department of Applied Chemistry \\ College of Science, China Agricultural University \\ Beijing 100193, P. R. China \\ †Yunnan Tobacco Company Dali Branch \\ Yunnan 671000, P. R. China \\ *Beijing Research Center for Agri-Food Testing \\ and Farmland Monitoring, Beijing 100097, P. R. China \\ $\S_{\text {wangd@nercita.org.cn }}$ \\ 『minsg@cau.edu.cn
}

Received 15 July 2013

Accepted 30 September 2013

Published 27 January 2014

\begin{abstract}
In this research, suitable imaging methods were used for acquiring single compound images of biology samples of chicken pectorales tissue section, tobacco dry leaf, fresh leaf and plant glandular hair, respectively. The adverse effects caused by the high water content and the thermal effect of near infrared (NIR) light were effectively solved during the experiment procedures and the data processing. PCA algorithm was applied to the NIR micro-image of chicken pectorales tissue. Comparing the loading vector of PC3 with the NIR spectrum of dry albumen, the information of PC3 was confirmed to be provided mainly by protein, i.e., the 3rd score image represents the distribution trend of protein mainly. PCA algorithm was applied to the NIR microimage of tobacco dry leaf. The information of $\mathrm{PC} 2$ was confirmed to be provided by carbohydrate including starch mainly. Compared to the 2nd score image of tobacco dry leaf, the compared correlation image with the reference spectrum of starch had the same distribution trend as the 2nd score image. The comparative correlation images with the reference spectra of protein, glucose, fructose and the total plant alkaloid were acquired to confirm the distribution trend of these compounds in tobacco dry leaf respectively. Comparative correlation images of fresh leaf with the reference spectra of protein, starch, fructose, glucose and water were acquired to confirm
\end{abstract}

\Corresponding author.

This is an Open Access article published by World Scientific Publishing Company. It is distributed under the terms of the Creative Commons Attribution 3.0 (CC-BY) License. Further distribution of this work is permitted, provided the original work is properly cited. 
the distribution trend of these compounds in fresh leaf. Chemimap imaging of plant glandular hair was acquired to show the tubular structure clearly.

Keywords: Near-infrared spectra micro-image; principal component analysis; compound distribution; tobacco leaf; plant glandular hair.

\section{Introduction}

In recent years, the chemical imaging (CI) analysis of biology sample has become a new focus in the field of analytical chemistry. However, problems, such as high water content, irregular shape, interferences, exist in imaging analysis of biology samples. Furthermore, the in situ analysis is also difficult in the CI analysis of biology samples.

Near-infrared spectra micro-imaging (abbreviated NIR micro-imaging) is one kind of $\mathrm{CI},{ }^{1}$ which is a new kind of technology in recent years with a lot of advantages. Being composed of the total absorbance values of the pixels in it, traditional IR imaging would only, to some extent, provide the comprehensive information of the analyzed object. However, it is unable to analyze the object from spectroscopic perspective. Being different from the traditional IR imaging, each pixel in the NIR spectra micro-image is a whole NIR spectrum. Not only can NIR spectra micro-imaging provide the spatial distribution information but also the spectroscopy information. As NIR beam has strong penetration ability, the samples whose thickness is nearly hundreds of micrometers can be scanned. Furthermore, NIR micro-imaging is one kind of nondestructive analysis method, which is suitable for the in situ analysis of fresh samples directly. In addition, quartz or glass can be used in the NIR micro-imaging experiment, which is relatively cheap and convenient to be cleaned. All these features mentioned above are superior to MIR micro-imaging technology. Meanwhile, NIR micro-imaging is free of strong florescence interferes. The imaging area can be as large as several square centimeters. Rough surface is also allowed to collect NIR microimage. All these features mentioned above are superior to Raman imaging. The NIR microscope can focus the NIR beam to several micrometers, which can enhance SNR efficiently. It is easy for NIR microscope to select tissue area; therefore, isolation for tissue cells is unnecessary, i.e., the in situ analysis would come true. The imaging quality would not be decreased even when ordinary objective lens are used in NIR band in the experiment of NIR micro-imaging.

Therefore, NIR micro-imaging would be a new method in the research of iconology and compounds distribution of fresh tissue and so forth, which would provide new information. NIR micro-imaging technology is fairly new in recent years. ${ }^{2}$ However, being different from MIR micro-imaging, for instance, imaging under a single wavelength (or peak) absorption, which is commonly used in MIR micro-imaging analysis, cannot be used in NIR micro-imaging in general for the reason of serious overlap of NIR spectrum. It is reported that NIR microscope has been applied to the spectra collection, ${ }^{3}$ the morphologic observation of microorganism or tissue, ${ }^{4,5}$ quantity tracking study ${ }^{6}$ and the morphology research of inorganic chemicals. ${ }^{7,8}$ However, all the applications mentioned above are not the single compound imaging strictly. Deriving the frequency doubling and combination band of molecular vibration of hydric groups, NIR spectra are usually overlapped seriously. Thus, it can be seen that the acquirement of single compound imaging from NIR micro-image is very difficult. There are few reports on the spectra separation algorithm applied in the analysis of NIR micro-imaging. There is hardly any report on the imaging analysis of biology samples by spectra separation algorithm.

In particular, there are mainly two difficulties in the analysis of NIR micro-image. In the first place, the high water content in the fresh biology samples would bring about strong absorption in NIR spectra, which would influence the analysis result seriously. In addition, fresh biology samples under the NIR light would dehydrate seriously in a short time, therefore, the samples would move out of focus. In other words, the images would become indistinct.

Therefore, the acquirement of both high-quality NIR micro-images and single compound imaging would be regarded as the objective in the field of NIR micro-imaging analysis. In this research, NIR 
micro-images of chicken pectorales tissue section and plant samples were collected. The originalities of this research would be reflected in the following two aspects: during the collection procedure, polystyrene film was used for preventing the dehydration or crispation caused by the thermal effect of NIR light; chemometrics algorithms having been applied to the information extraction from NIR micro-images of biology samples. Single compound chemical images were acquired by spectra separation algorithm effectively. This research would, to some extent, provide reference to the spatial distribution analysis of chemical compounds in biology samples.

\section{Research Methodology}

\subsection{Samples}

(1) Chicken pectorales tissue section. In order to make the chicken pectorales tissue hard to make section, the chicken pectorales tissue was embedded by the embedding medium of paraffins. The section would be suitable for observation by optical microscope. Hematoxylineosin staining method, which is the basic and frequently used method in histology, embryology, pathology and so forth, was applied to stain the pectorales tissue in the section. After the pectorales tissue was stained, the cytoplasm with the main compound of protein was stained red.

(2) Tobacco dry leaf sample. A small piece of flat area of the flue-cured tobacco leaf was cut off to stick on the quartz glass slide with a very thin piece of polystyrene film in order to prevent the crispation and out of focus caused by the thermal effect of the NIR light. The main chemical compounds of tobacco dry leaf are carbohydrate and plant alkaloid (nicotine in major).

(3) Fresh leaf sample. A piece of fresh leaf from the broad leaf pot plant was acquired, a small piece was cut to stick on the quartz glass slide with a very thin piece of polystyrene film in order to prevent the dehydration and out of focus caused by the thermal effect of NIR light. The main chemical compounds of fresh leaf are water and carbohydrate.

(4) Plant glandular hair sample. A fresh leaf of pumpkin with glandular hair was acquired, the leaf tissue with glandular hair was cut off to stick on the quartz glass slide with a very thin piece of polystyrene film in order to prevent the dehydration and out of focus caused by the thermal effect of NIR light. The main chemical compounds of fresh leaf are water and carbohydrate with carbon-hydrogen skeleton structure.

\subsection{Instrument}

In this research, the NIR micro-images were collected by FT-NIR Micro-Image System (Version: Spotlight 350, Perkin Elmer, US) with the optical souse of halogen-tungsten lamp and the liquid nitrogen cooled mercury-cadmium-telluride (MCT) detector.

The NIR micro-images were collected as follows. The area on the slide without sample was regarded as the reference so as to deduct the background effect, the micro-images of samples were collected under the transmission mode. Taking the tobacco dry leaf sample for example, the reference is selected at the quartz glass slide with polystyrene film but without tobacco dry leaf.

Instrument parameters are described as follows. Wavenumber range for collection: 7800-4000 $\mathrm{cm}^{-1}$, while the wavenumber for imaging analysis would be selected according to the different characteristics of the samples. Spectral resolution and spatial resolution would be selected according to the physicochemical property and the complexity of chemical compounds. The high spectral resolution is suitable for samples with simple chemical compounds which would have relatively sharp absorption peaks, while the high spatial resolution is suitable for samples with microscopic spatial structures. In this research, the spectral resolution of $16 \mathrm{~cm}^{-1}$ is suitable for chicken pectorals tissue section sample, $64 \mathrm{~cm}^{-1}$ for tobacco dry leaf sample and fresh leaf sample, $32 \mathrm{~cm}^{-1}$ for plant glandular hair sample; while the spatial resolution of $6.25 \mu \mathrm{m} \times 6.25 \mu \mathrm{m}$ is suitable for chicken pectorales tissue section sample and plant glandular hair sample, $25 \mu \mathrm{m} \times 25 \mu \mathrm{m}$ for tobacco dry leaf sample and fresh leaf sample.

\subsection{Imaging methods}

The frequently used imaging algorithm in the analysis of NIR micro-imaging are compared with correlation imaging, chemimap imaging, principal component analysis score imaging (PCA-score imaging) and so forth. Comparative correlation imaging 


\section{Wang et al.}

is reconstructed according to NIR micro-image data structure by the correlation coefficients between the spectra in NIR micro-image and the reference spectrum. For the comparative correlation imaging, the highlight pixels represent higher similarity between these pixels' spectra and the reference spectrum, while the lowlight ones represent lower similarity. Chemimap imaging is composed by the integral values of the feature peaks or bands of target compound. For the CI, the highlighted pixels represent that the spectra of these pixels have stronger chemical feature, while the lowlight ones represent weaker chemical feature.

Principal component analysis (PCA) score imaging would be able to separate the spectra by the extraction of the eigen vectors of the NIR microimage data by PCA algorithm, which would decompose the raw data matrix into several orthogonal eigen vectors (loadings) with score matrix in which the vectors are the linear combination of the raw data. However, the reconstructed data matrix by these loadings and scores would be able to characterize the original data feature and structure without eliminating the useful information. PCAscore imaging is composed of the score values of PCA according to spatial structure of NIR microimage data, the meaning of which could be interpreted by the corresponding eigen vector, i.e., the loading.

\section{Result and Discussion}

\subsection{Analysis of NIR micro-image of chicken pectorals tissue section}

The chicken pectorales tissue section was regarded as the research object to acquire NIR micro-image. The wavenumber range for imaging analysis is $5500-4000 \mathrm{~cm}^{-1}$, which is the combined frequency absorption area of protein mainly. The visible image of chicken pectorales tissue is shown in Fig. 1(a), while the NIR total absorption micro-image is shown in Fig. 1(b). Since the section was stained by HE staining method, the residual on the section is mainly the protein in the cytoplasm.

The chicken dry albumen was regarded as protein mainly in this research. In order to extract the main compound information of chicken pectorales tissue and acquire the single compound imaging, PCA algorithm was applied to the analysis of NIR micro-image of chicken pectorales tissue. A total of

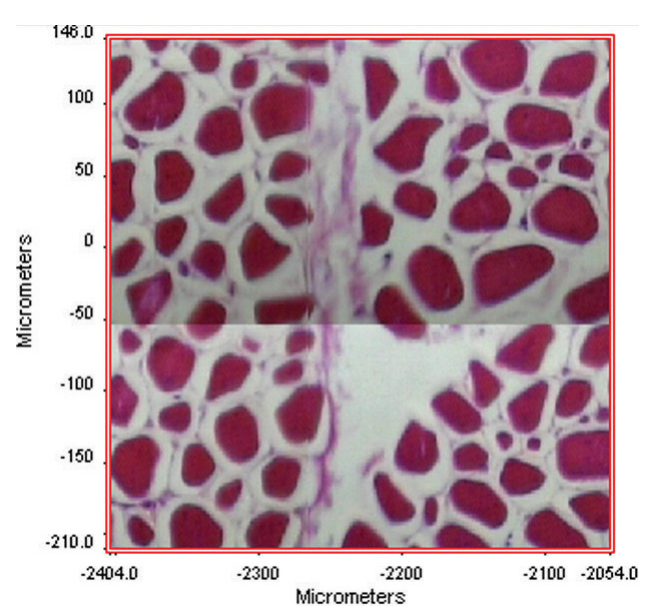

(a)

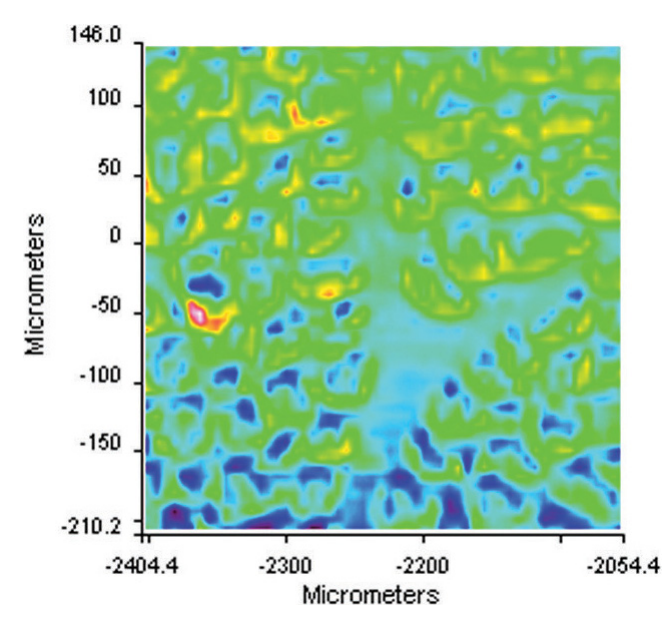

(b)

Fig. 1. Visible image (a) and NIR total absorption microimage (b) of chicken pectorales tissue.

20 principal components were extracted after PCA. It could be seen from Fig. 2(a) that similar data features exist at $5157 \mathrm{~cm}^{-1}$ (P1), $4866 \mathrm{~cm}^{-1}$ (P2) and $4600 \mathrm{~cm}^{-1}$ (P3) between the loading vector of PC3 [see Figs. 2(a)-2(II)] and the NIR spectrum of dry albumen [see Figs. 2(a)-2(I)], which is also the NIR features of protein. However, it is different between the two data groups during the wavenumber range of $4500-4000 \mathrm{~cm}^{-1}$, which derives from the fat in chicken dry albumen for the NIR spectrum of dry albumen [see Figs. 2(a)-2(I)]. This result indicated that the information of PC3 is mainly provided by protein, but not other compounds. Score image of PC3 [see Fig. 2(b)] represented the distribution trend of protein mainly, which had the same distribution trend as the visible image of chicken pectorales tissue [see Fig. 1(a)]. 


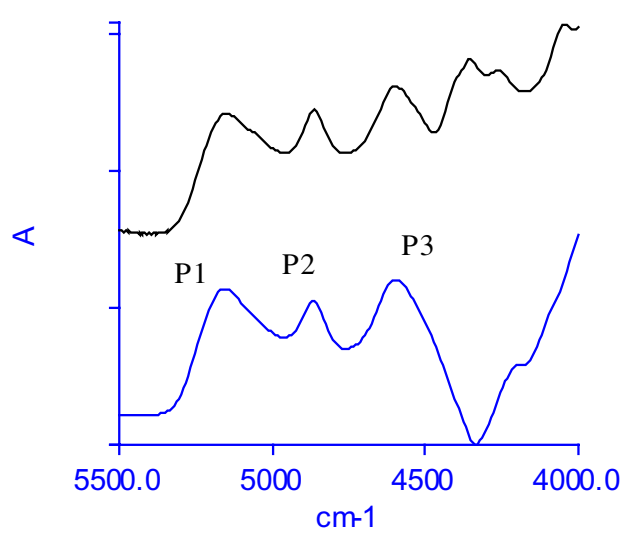

(a)

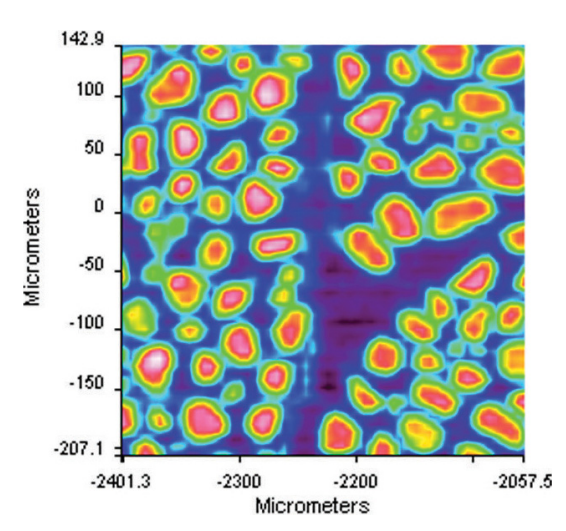

(b)

Fig. 2. NIR spectrum of dry albumen (a)-(I), PC3 eigen vector (a)-(II) and the 3rd score image (b).

The analysis indicated that PCA would extract single compound feature of protein in chicken pectorals tissue from NIR micro-image. In this research, PC3 loading vector and NIR spectrum of dry albumen that mainly characterizes protein was compared, it could be determined that the information of PC3 derives from protein mainly, i.e., score imaging of $\mathrm{PC} 3$ represents the distribution trend of protein mainly, which is consistent with the practical situation.

\subsection{Analysis of NIR micro-image of plant tissues}

\subsubsection{Analysis of NIR micro-image of tobacco dry leaf}

A piece of tobacco dry leaf was put on a clean object slide, which was regarded as the research object to acquire NIR micro-image. The wavenumber range

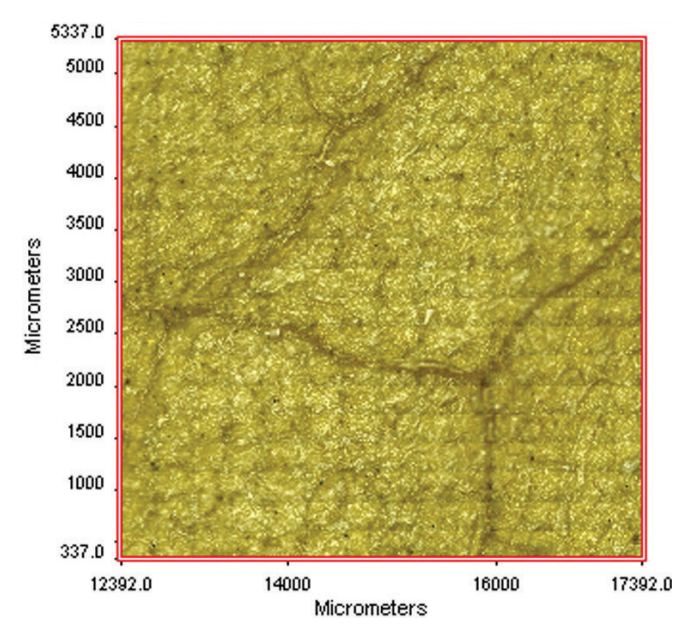

(a)

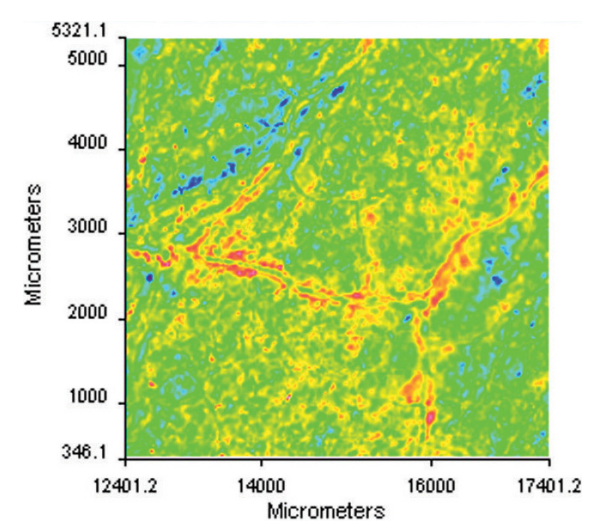

(b)

Fig. 3. Visible image (a) and NIR total absorption image (b) of tobacco dry leaf.

for analysis is $7680-4000 \mathrm{~cm}^{-1}$, which is as wide as the imaging system could collect. Therefore, the whole spectral information would be used. The visible image is shown in Fig. 3(a), while the NIR total absorption micro-image is shown in Fig. 3(b).

PCA algorithm was applied to the analysis of NIR micro-image of tobacco dry leaf in order to extract the single compound information. As is shown in Fig. 4, similar data features exist between the two data groups of $\mathrm{PC} 2$ eigen vector [see Fig. 4(a)] and NIR spectrum of starch [see Fig. 4(b)], while the correlation coefficient between the two data groups is 0.9779 . Therefore, it could be confirmed that the information of $\mathrm{PC} 2$ is provided by carbohydrate including starch mainly and the 2nd score image [see Fig. 4(c)] would represent the distribution trend of carbohydrate (saccharides) including starch mainly. 
D. Wang et al.

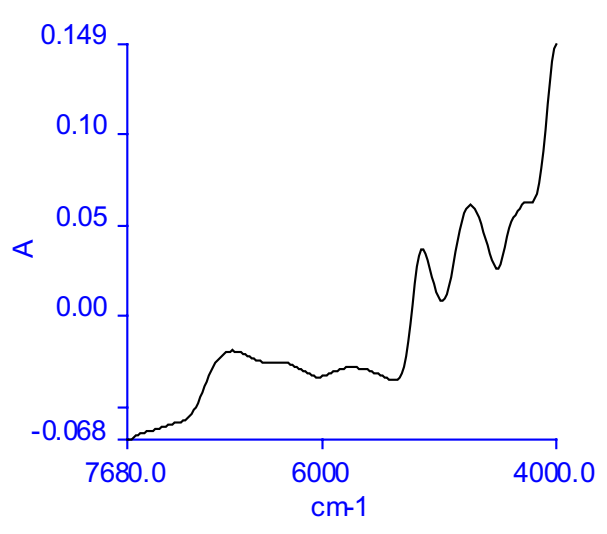

(a)

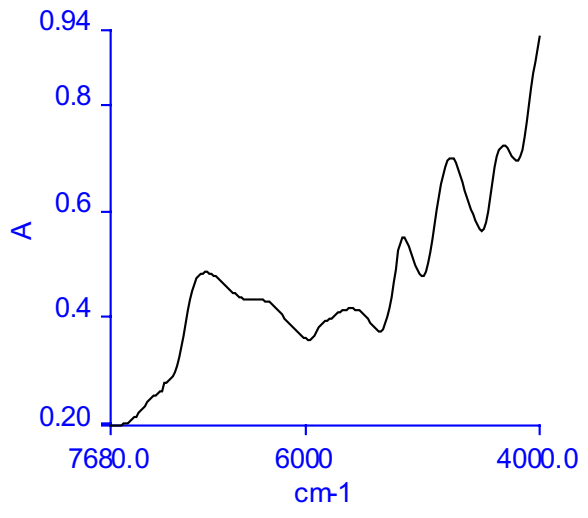

(b)

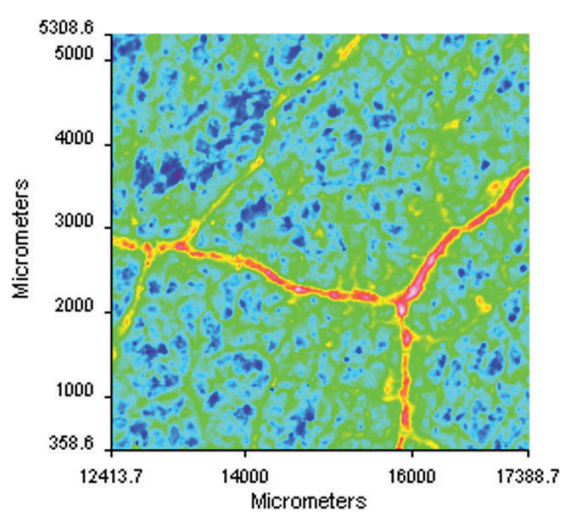

(c)

Fig. 4. PC2 eigen vector (a), NIR spectrum of starch (b) and the 2nd score image (c).

Comparative correlation imaging is an intuitionistic imaging algorithm with relative simple calculation process, which is also suitable for the imaging analysis of the complicated samples. For the tobacco dry leaf sample, comparative correlation imaging with the reference spectrum of starch was acquired. Comparative correlation imaging was applied to the analysis of NIR micro-image of tobacco dry leaf with the reference spectrum of starch, which is shown in Fig. 5(b). Upon comparison, Figs. 4(c) and 5(b) had the same distribution trend. This result indicated that comparative correlation imaging would be able to acquire the single compound imaging of tobacco dry leaf. According to that, the compare correlation images with the reference spectra of protein, starch, fructose, glucose and the total plant alkaloid (nicotine in major) were acquired respectively, which is shown in Fig. 5 . It could be seen from Fig. 5 that the five compounds in the tobacco dry leaf are concentrated in leaf veins mainly. The reason is that leaf veins have the tubular structure, which is relatively hard than tobacco leaf cells. The leaf veins operate on skeletons and the carriage of chemical compounds. The tobacco leaf cells would dehydrate during the flue-cured procedures. Therefore, the compounds in leaf cells would be transferred into leaf veins whose interspaces are relatively larger.

The analysis result of Fig. 5 indicated that comparative correlation imaging is a convenient method to show the distribution trends of protein, carbohydrate (saccharides), the total plant alkaloid and so forth on NIR micro-image of tobacco dry leaf.

\subsubsection{Analysis of NIR micro-image of fresh leaf}

A piece of fresh leaf was put on a clean object slide, which was regarded as the research object to acquire NIR micro-image. In order to avoid the adverse effect of water in fresh leaf, the wavenumber range for analysis is $6000-4000 \mathrm{~cm}^{-1}$, which is mainly the combined frequency band with stronger spectral 


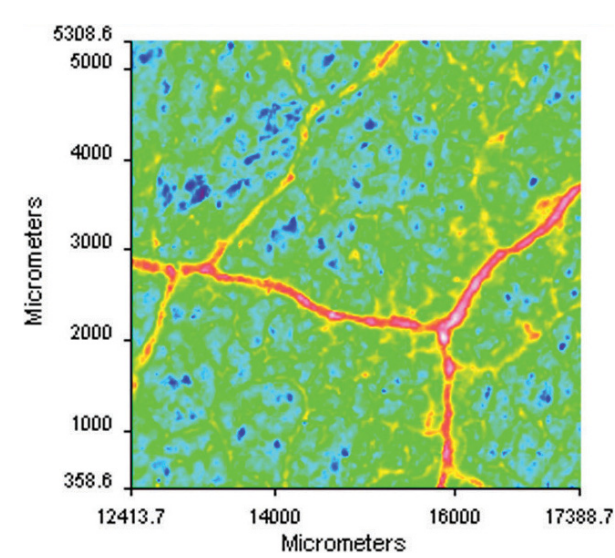

(a)

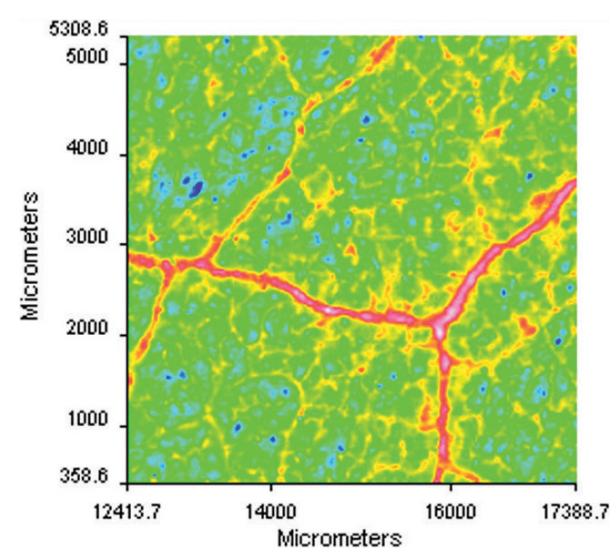

(c)

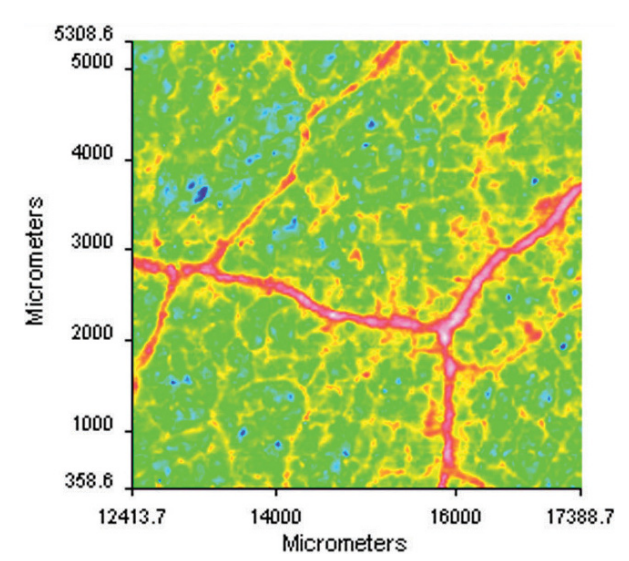

(b)

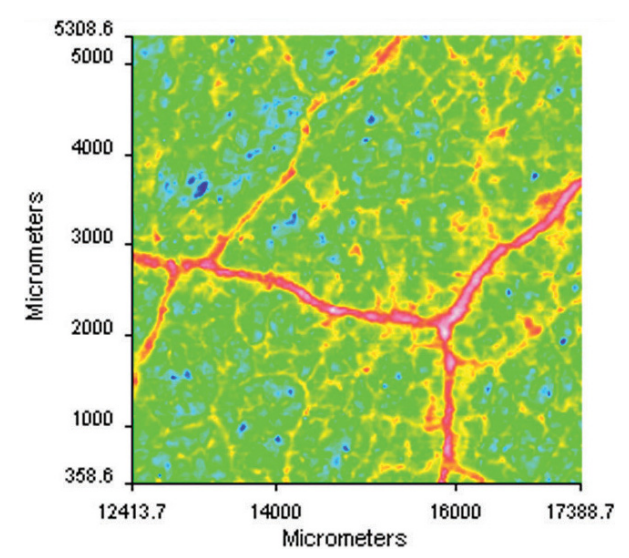

(d)

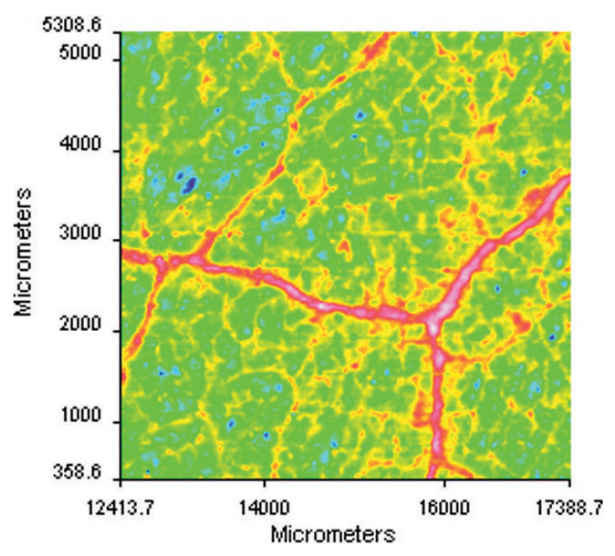

(e)

Fig. 5. Comparative correlation images of tobacco dry leaf: (a) protein, (b) starch, (c) fructose, (d) glucose and (e) the total plant alkaloid.

information. The visible image is shown in Fig. 6(a), while the NIR total absorption micro-image is shown in Fig. 6(b). The distribution trend of leaf veins in fresh leaf can be seen from Figs. 6(a) and 6 (b) directly.
Comparative correlation images of tobacco dry leaf having been regarded as reference, comparative correlation images of fresh leaf were acquired with the reference spectra of protein, starch, fructose, glucose and water, respectively, which are shown in 
D. Wang et al.

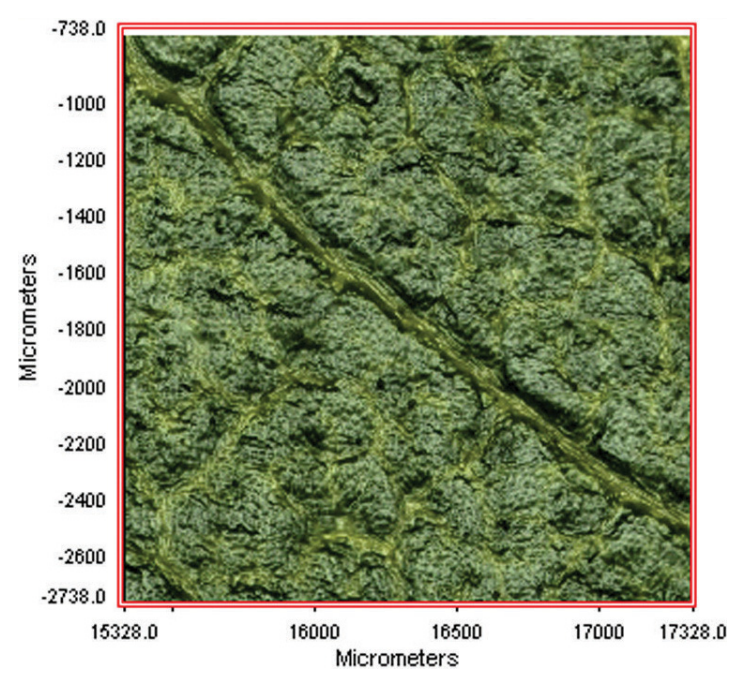

(a)

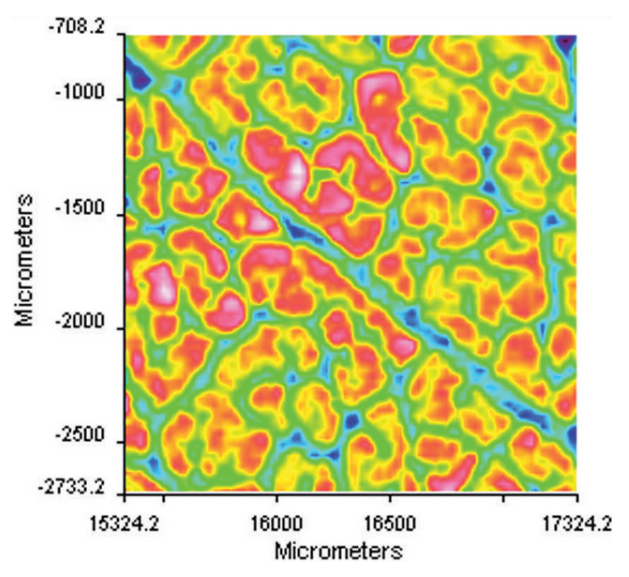

(b)

Fig. 6. Visible image (a) and NIR total absorption image and (b) of fresh leaf.

Fig. 7. It could be seen that the five compounds are concentrated mainly in leaf cells. The reason is that the compounds in fresh leaf come from leaf cells mainly. As shown in Fig. 7(e), the distribution trend of water in either leaf cells or leaf veins is nearly the same.

\subsubsection{Analysis of the NIR micro-image of plant glandular hair}

The plant (pumpkin leaf) glandular hair was regarded as a research object to acquire NIR microimage. Visible image and NIR total absorption micro-image are shown in Figs. 8(a) and 8(b), respectively. Due to the tubular structure of plant glandular hair, being observed from side face, both sides of the glandular hair with more organic compound, i.e., carbohydrate with carbon-hydrogen skeleton structure, are relatively thicker than the middle part of it, which could be proved by NIR total absorption micro-image approximately. There is high absorption in the two sides of the glandular hair while there is low absorption in the middle part of it.

For further proof of the viewpoint mentioned above, chemimap imaging with the wavenumber range of $4200-4000 \mathrm{~cm}^{-1}$ was applied to this example, of which the wavenumber range was the combined tone of $\mathrm{C}-\mathrm{H}$ group mainly. The chemimap imaging of plant glandular hair is shown in

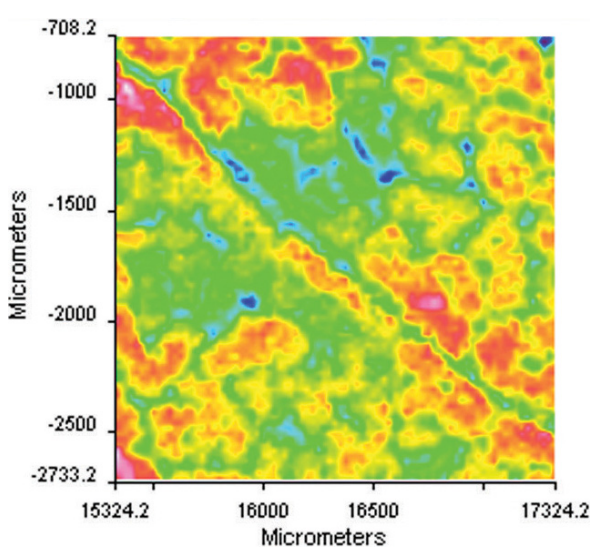

(a)

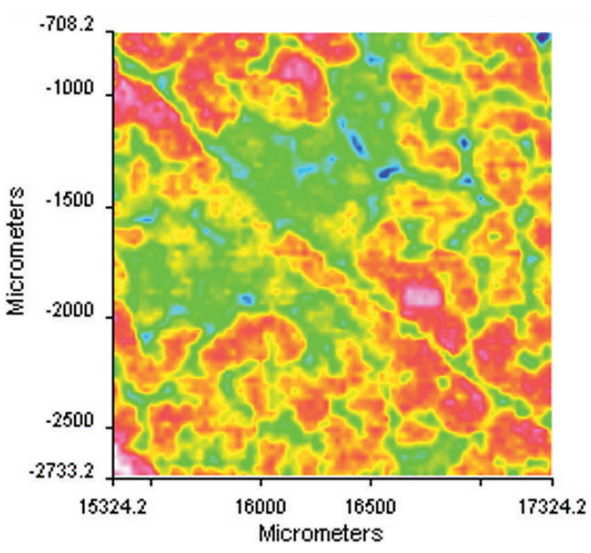

(b)

Fig. 7. Comparative correlation images of fresh leaf: (a) protein, (b) starch, (c) fructose, (d) glucose, (e) water. 
The Application of NIR Spectra Micro-Image in the Imaging Analysis of Biology Samples

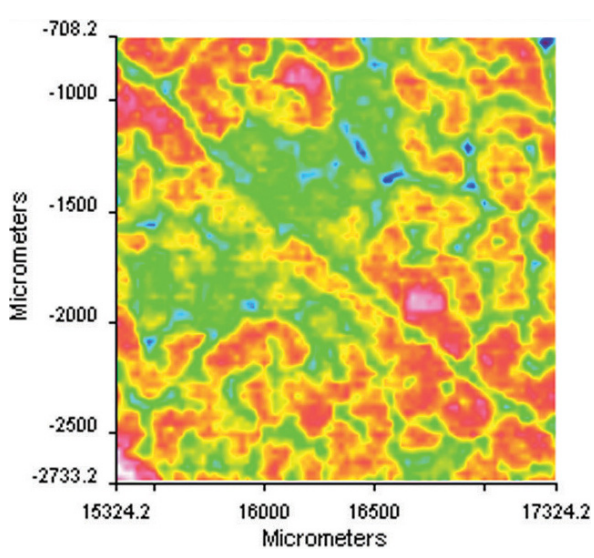

(c)

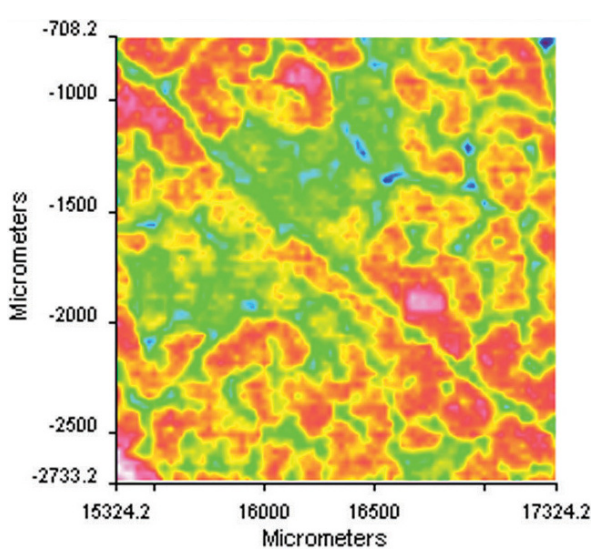

(d)

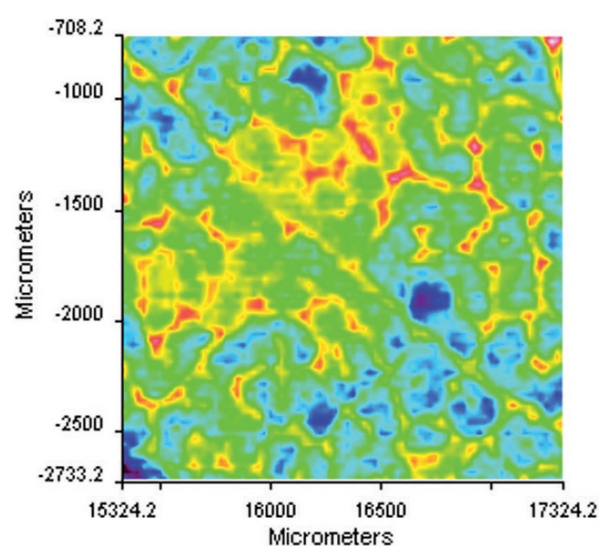

(e)

Fig. 7. (Continued)

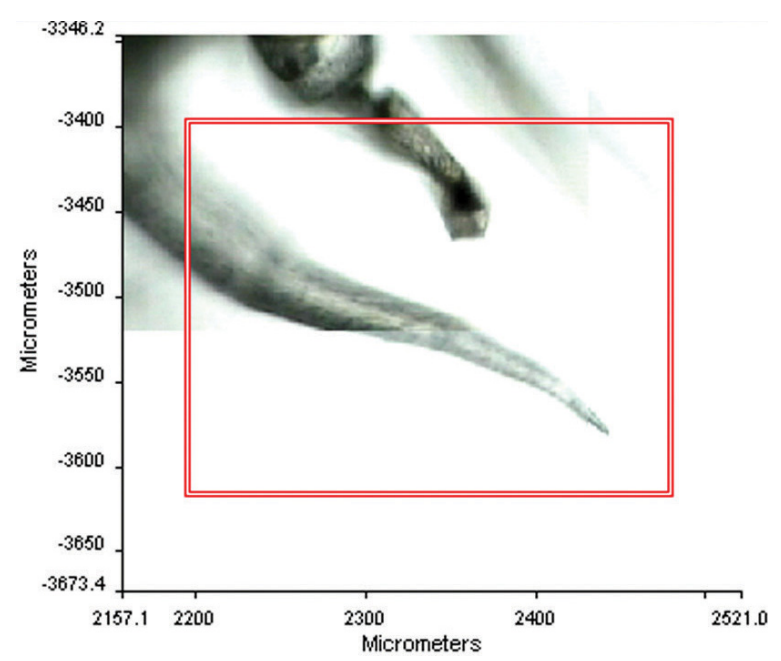

(a)

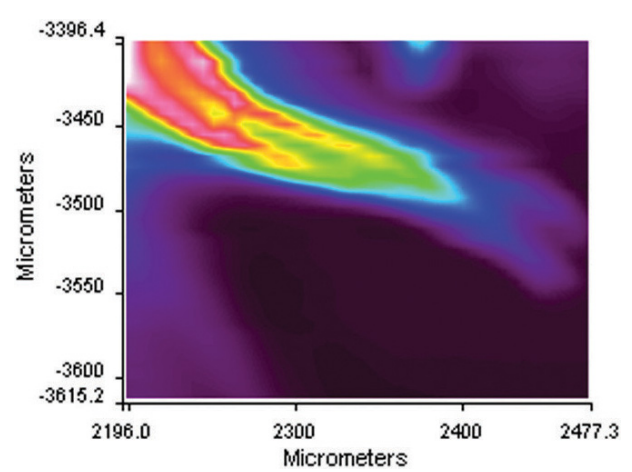

(b)

Fig. 8. Visible image (a) and NIR micro-image (b) of the plant glandular hair. 


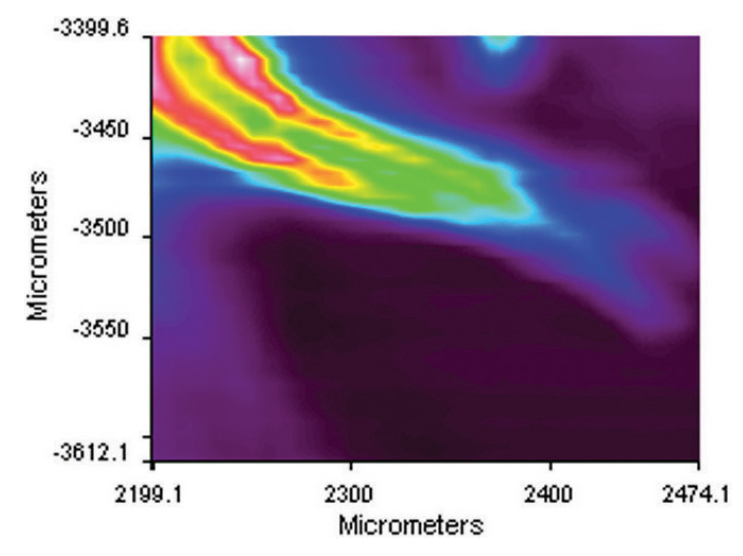

Fig. 9. Chemimap imaging of the plant glandular hair.

Fig. 9. It could be seen from Fig. 9 that chemimap imaging is able to show the tubular structure of plant glandular hair more clearly.

\section{Conclusion}

In this research, the biology samples of chicken pectorales tissue section, tobacco dry leaf, fresh leaf and plant glandular hair were regarded as research objects, of which the NIR micro-images were collected. During the experiment procedure, polystyrene film was used for preventing the deformation caused by dehydration under thermal effect of NIR light. Chemometrics algorithms, such as principal component analysis, comparative correlation imaging and chemimap imaging, were applied in extracting the single compound information from NIR micro-images, single compound images were acquired successfully. The result indicated that NIR micro-imaging technology would be widely applied in the field of biology research with the advantages of nondestruction and in situ analysis, especially the confirmation of the spatial distribution of chemical compounds simultaneously. However, superior imaging methods applied in the analysis of NIR micro-image to acquire the spatial distribution trends of more compounds more efficiently are still being researched.

\section{Acknowledgments}

This study is supported by the National Natural Science Foundation of China (No. 20575076). This paper was supported by the National Natural Science Foundation of China [No. 20575076].

\section{References}

1. A. A. Gowen, C. P. O'Donnell, P. J. Cullen, S. E. J. Bell, "Recent applications of chemical imaging to pharmaceutical process monitoring and quality control," Eur. J. Pharm. Biopharm. 69, 10-22 (2008).

2. F. Clarke, "Extracting process-related information from pharmaceutical dosage forms using near infrared microscopy," Vibrational Spectrosc. 34, 25-35 (2004).

3. B. de la Roza-Delgado, A. Soldado, A. MartínezFernández, F. Vicente, A. Garrido-Varo, D. PérezMarn, M. J. de la Haba, J. E. Guerrero-Ginel, "Application of near-infrared microscopy (NIRM) for the detection of meat and bone meals in animal feeds: A tool for food and feed safety," Food Chem. 105, 1164-1170 (2007).

4. J. Dubois, E. N. Lewis, F. S. Fry Jr., E. M. Calvey, "Bacterial identification by near-infrared chemical imaging of food-specific cards," Food Microbiol. 22, 577-583 (2005).

5. Z. S. Tannous, M. C. Mihm, T. J. Flotte, S. González, "In vivo examination of lentigo maligna and malignant melanoma in situ, lentigo maligna type by nearinfrared reflectance confocal microscopy: Comparison of in vivo confocal images with histologic sections," Dermatopathology 46(2), 260-263 (2002).

6. E. J. Roy, M. Sivaguru, G. Fried, B. D. Gray, D. M. Kranz, "Imaging membrane intercalating near infrared dyes to track multiple cell populations," J. Immunol. Methods 348, 18-29 (2009).

7. F. J. Rios, J. V. Alves, C. A. Pérez, É. C. Costa, C. A. Rosière, K. Fuzikawa, J. M. C. Neves, A. de O. Chaves, S.P. Prates, R. E. de Barrio, "Combined investigations of fluid inclusions in opaque ore minerals by NIR/ SWIR microscopy and microthermometry and synchrotron radiation X-ray fluorescence," Appl. Geochemistry 21, 813-819 (2006).

8. Y. Inatomi, M. Kikuchi, R. Nakamurac, K. Kuribayashi, I. Jimbob, "In situ observation for semiconductor solution growth using a near-infrared microscope," J. Cryst. Growth 275, 193-200 (2005). 